\title{
Performance of statistical methods to correct food intake distribution: comparison between observed and estimated usual intake
}

\author{
Eliseu Verly-Jr ${ }^{1 *}$, Dayan C. R. S. Oliveira ${ }^{1}$, Regina M. Fisberg ${ }^{2}$ and Dirce Maria L. Marchioni ${ }^{2}$ \\ ${ }^{1}$ Institute of Social Medicine, Rio de Janeiro State University, Rio de Janeiro, 20550-013, Brazil \\ ${ }^{2}$ School of Public Health, University of São Paulo, São Paulo, 01246-904, Brazil \\ (Submitted 2 June 2015 - Final revision received 29 February 2016 - Accepted 23 June 2016 - First published online 2 August 2016)
}

\section{Abstract}

There are statistical methods that remove the within-person random error and estimate the usual intake when there is a second 24-h recall (24HR) for at least a subsample of the study population. We aimed to compare the distribution of usual food intake estimated by statistical models with the distribution of observed usual intake. A total of 302 individuals from Rio de Janeiro (Brazil) answered twenty, non-consecutive $24 \mathrm{HR}$; the average length of follow-up was 3 months. The usual food intake was considered as the average of the 20 collection days of food intake. Using data sets with a pair of 2 collection days, usual percentiles of intake of the selected foods using two methods were estimated (National Cancer Institute (NCI) method and Multiple Source Method (MSM)). These estimates were compared with the percentiles of the observed usual intake. Selected foods comprised a range of parameter distributions: skewness, percentage of zero intakes and within- and between-person intakes. Both methods performed well but failed in some situations. In most cases, NCI and MSM produced similar percentiles between each other and values very close to the true intake, and they better represented the usual intake compared with 2-d mean. The smallest precision was observed in the upper tail of the distribution. In spite of the underestimation and overestimation of percentiles of intake, from a public health standpoint, these biases appear not to be of major concern.

Key words: Usual intakes: Measurement errors: Food consumption: Within-person variance

In nutritional epidemiology, the usual dietary intake of individuals is the relevant exposure for investigating dietdisease relationships properly. Usual dietary intake is defined as the long-run average of daily intakes ${ }^{(1)}$. When compared with long-term, self-report instruments to collect dietary intake, short-term instruments such as 24-h recalls (24HR) and food records are believed to have lower systematic error, usually presenting a lower underestimation of energy and protein intakes ${ }^{(2)}$. Nonetheless, they are highly affected by day-to-day variation - that is, the within-person variation in dietary intake in that a single collection day is not sufficient to reproduce the usual food intakes. The implications of using information of intake from an instrument with this kind of error (called withinperson random error in this case) are a biased estimate of the intake distribution, inflated variance, percentiles severely underestimated and overestimated, and proportions of a population above some cut-off point wrongly estimated ${ }^{(3,4)}$. Considering that administering several $24 \mathrm{HR}$ per individual in large epidemiological studies is difficult because of time consumption, cost and burdensome respondents, statistical methods such as the National Cancer Institute $(\mathrm{NCI})^{(5)}$ method and Multiple Source Method (MSM) ${ }^{(6)}$ were developed to remove the within-person random error and to estimate the usual intake when there is a second $24 \mathrm{HR}$ for at least a subsample of the study population. In fact, the use of statistical methods to correct percentiles of intake by removing the effect of the within-person variance has been implemented and recommended in many studies. Since 2003, the National Health and Nutrition Examination Survey has collected a replication of the $24 \mathrm{HR}$ in a subsample to allow estimation of the usual dietary intake. In Brazil, these methods have been implemented in most of the studies using data from national representative studies ${ }^{(7)}$. Most recently, the WHO supported the use and distribution of Intake Monitoring, Assessment and Planning Program software based on similar statistical models.

Simulation studies have shown good performance of these methods in estimating dietary items that are consumed every day or almost every day by most people (which is the case of energy and most nutrients) ${ }^{(8)}$, but have not always been satisfactory when estimating usual intake of items consumed episodically (which is the case of foods) ${ }^{(9)}$. In these studies, data simulation was used to obtain a true dietary intake for each individual, and then used to compare with the estimates from those methods. Although simulation studies can often be considered a good representation of real life, they are used as surrogates of real data. In the dietary intake context, where

Abbreviations: 24HR, 24-h recalls; MSM, Multiple Source Method; NCI, National Cancer Institute.

* Corresponding author: E. Verly-Jr, email eliseujunior@gmail.com 
there is a complex correlation within and between individuals and food items, simulated data may not adequately reproduce a real scenario ${ }^{(10)}$. In this sense, using real data that provide usual dietary intake is preferable to assess the performance of these models. The VIESP study, which stands for 'intrapersonal variance of dietary intake and bias in nutritional epidemiological studies' in Portuguese, has measured usual food and nutrient intakes in a sample by administering twenty, nonconsecutive $24 \mathrm{HR}$ in each individual. Thus, the aim of this study was to compare the distribution of usual food intake estimated by statistical models with the distribution of the observed usual intake. The results will allow us to have a critical discussion regarding both conclusions of studies that adopted these methods and the recommendation for use in future studies.

\section{Methods}

\section{Study design and population}

The present study was a longitudinal study including 302 individuals from the city of Rio de Janeiro, Brazil. To maintain both quality and participation rate, as well as for logistic and cost reasons, we adopted a snowball sampling: we first selected the interviewers (twenty-three undergraduate dietitians) and they in turn selected individuals to administer the $24 \mathrm{HR}$. These selected participants preferably frequented the same social network, were close to the interviewer and were willing to answer in detail about everything they consumed for 20 non-consecutive days. Although the interviewers were from the same university, the respondents were well spread throughout the city, as each interviewer had his or her own social network, that is, each one lived in a different neighbourhood, with no common friends and relatives with other interviewees. Data collection was performed from March 2013 to April 2014 and covered weekdays and weekend days. The average length of follow-up for each individual was 3 months. The interviewers were warned not to administer the $24 \mathrm{HR}$ on the same weekdays for a given individual.

\section{Dietary assessment}

The multiple-pass method for intake collection by $24 \mathrm{HR}$ was applied by trained interviewers. This method uses distinct steps to collect information about the participant's food intake, including listing everything consumed the previous day, the time of consumption, descriptions about and amounts of each food reported and review of the list of foods mentioned and probes for additional eating occasions ${ }^{(11)}$. The first $24 \mathrm{HR}$ by the interviewers were checked by the research staff. In case of incomplete filling, the interviewer was re-trained with respect to their specific mistakes. After this initial checking, interviewers were asked to periodically submit the filled $24 \mathrm{HR}$ to the research staff for quality check. Information on food intake was entered using software specifically designed for Brazilian dietary surveys (Brasil Nutri), which contained standardised portions for foods and recipes most commonly found in the national representative household purchase research carried out in 2002-2003. As the 24HR were being entered, a research assistant performed a screen out to detect and correct mistyped records and inconsistent amounts coded. Foods reported in each $24 \mathrm{HR}$ were critically reviewed to identify any failures related to the descriptions of the food consumed or to food-preparation techniques, including their apportioning and quantification.

\section{Statistical analysis}

True usual food intake was defined as the average of the twenty 24HR for each individual. This was considered our 'gold standard' for usual dietary intake for further comparisons and analyses. Usual food intakes can be estimated by statistical models using two non-consecutive $24 \mathrm{HR}$ for each individual $^{(5,6)}$. However, as the sample size was not large enough, the precision of percentiles of intake was lower, so that the estimation using, for instance, the 1 st and 2nd collection days would be different from that using the 1st and 3rd, from that using the 4 th and 7 th and so on. To overcome this limitation, we generated data sets containing all possible combinations of collection days - that is, one with the 1 st and 2 nd collection days, then the 1 st and $3 \mathrm{rd}$, then the 1 st and $4 \mathrm{th}, \ldots$, the 2 nd and $3 \mathrm{rd}$, the 2nd and 4th and so on till the 19th and 20th collection days - totalling up to 190 data sets. For each combination, we estimated usual percentiles of intake for the selected foods using two methods briefly described as follows.

- National Cancer Institute method ${ }^{(5)}$ : it is a two-part, mixed model in which the first part estimates the probability of consuming a food, and the second part estimates the amount consumed. Both parts include person-specific random effects that allow for correlation between probability and amount. Usual intake distribution is estimated on the basis of parameters defined in the model, such as within- and between-person variance, $\lambda$ of Box-Cox transformation and population mean. Percentiles of usual intake were estimated using MIXTRAN and DISTRIB macros version 2.1 (available on appliedresearch.cancer.gov) that run in SAS software package (SAS Institute Inc.).

- Multiple Source Method ${ }^{(6)}$ : MSM consists of a web-based software developed within the framework of the European Food Consumption Validation Project. It is also a two-part correlated model, but in this case parameters of the usual intake distribution are calculated directly from distribution of the estimated individual usual intake. The MSM method was accessed to perform the analyses on the website msm.dife.de. Detailed statistical properties of these methods can be accessed in another publication ${ }^{(9)}$.

We selected food items in which usual intake distribution could be well described by averaging 20 collection days and comprised a range of parameter distributions: skewness, CV, percentage of zero intakes, percentage of non-consumers, within:between-person variance ratio and the correlation between probability of intake and amount consumed. The online Supplementary Material shows detailed analyses on which the selection of foods was based. For each percentile of intake, we obtained 190 possible estimates (e.g. we have one estimate for the 1st percentile of each one of the 190 data sets). 
We then averaged these estimated percentiles to express the most expected estimate for this percentile of intake. To compare the distributions - estimated from NCI, estimated from MSM and 2-d mean - graphs with the Kernel density of the distributions and absolute difference were plotted. The precision of the estimation was expressed as the interval between the 5th and the 95th percentiles from the distribution of all possible estimates (i.e. 190) for a given percentile. Biases were calculated as the difference between the estimated and the true intake, and the lower and upper limits of the bias were the differences between the lower and the upper interval of the estimated percentiles and the true intake, respectively. Bias and their upper and lower limits were plotted to present precision of the estimated percentiles of intake using both methods. In addition, we fit both methods to all $20 \mathrm{~d}$ to assess potential improvement in using more than just one replication.

The study protocol was reviewed and approved by the Ethics Committee at the Institute of Social Medicine, State University of Rio de Janeiro. All individuals signed the informed consent form.

\section{Results}

The average age of the participants was 30 (SD 11) years; $71 \%$ of the sample were women; and 14, 25 and $61 \%$ of the sample had elementary, high school and college education, respectively. The percentages of interviews according to the days of the week were $16 \cdot 3,15 \cdot 1,16,14 \cdot 5,12 \cdot 2$ and $11 \cdot 2 \%$ for Sunday to Saturday, respectively.

Table 1 shows the mean, CV, skewness, percentage of zero intake, percentage of non-consumers, variance ratio (withinto-between-person variance) and the correlation between probability of intake and amount eaten for selected foods. In spite of a high percentage of zero intake in a given day of intake, the combined $20 \mathrm{~d}$ showed a small percentage of non-consumers with the exception for coffee (19\%). For most foods, there was an important correlation between probability of intake and amount eaten, which indicates the appropriateness of using the two-part correlated model.

Fig. 1(a)-(h) presents the distributions based on true intake, estimated from NCI and MSM, and the 2-d mean. In most cases, NCI and MSM produced similar percentiles that were very close to the true intakes, and they were better in representing the usual intake compared with 2-d mean. Fig. 2(a)-(h) shows the precision for each estimated percentile of intake for selected foods. The solid line and the shaded area stand, respectively, for the biases and precision of the MSM method, and circles and bars are the estimates for the NCI method. With a few exceptions, both NCI and MSM underestimated the intake in the upper percentiles, mainly from the 85th percentile onwards. Both NCI and MSM overestimated the low percentiles mainly for foods with high percentage of zero intake in a 2-d combination, which was the case for coffee, fruits, candies and chicken. Fig. 3(a) and (b) compare estimation using 2 and $20 \mathrm{~d}$ records with true intakes. NCI provided very similar estimates either using 2 or $20 \mathrm{~d}$ records; MSM estimations presented a higher variation mainly in the low percentiles for total meat.

\section{Discussion}

We demonstrated empirically that both methods provide good estimates of the usual food intake distribution but may fail in some situations. Both methods overestimated the low percentiles, specifically up to about the 15 th and 20 th percentile. This happened more intensively when estimated by MSM, with overestimation of the lower percentiles (please see percentiles up to 20th-30th for these foods) followed by a higher underestimation (please see percentiles from 30th to 40th). This is in part due to statistical properties of the methods: although NCI estimates percentiles of intake based on parameters defined from the population that produce a smoothed curve, the curve from MSM is based on estimated individual intake, being more similar to the shape of the original intake distribution.

Our results have important practical implications for dietary assessment in nutritional epidemiological studies once we provide quantitative evidence that distribution of usual food intake can be estimated using only one replication of the $24 \mathrm{HR}$, which can even be performed in a subsample of the study population $^{(12)}$. Moreover, there is no apparent advantage of fitting the models using more than one replication, mainly for the NCI model. In the case of MSM, it seems that adding more collection days would result in better estimates in low

Table 1. Parameter distribution for selected food items (Mean values and coefficients of variation)

\begin{tabular}{|c|c|c|c|c|c|c|c|}
\hline & Mean & CV\% & Skewness & $\begin{array}{l}\text { Percentage of } \\
\text { zero intakes }{ }^{*}\end{array}$ & $\begin{array}{c}\text { Percentage of } \\
\text { non-consumers } †\end{array}$ & VR & $\rho \ddagger$ \\
\hline Beans & 108.4 & 129 & 2.07 & 24 & 0 & 1.00 & 0.42 \\
\hline Coffee & $90 \cdot 2$ & 137 & 1.93 & 38 & 19 & 1.09 & 0.45 \\
\hline Soft drinks & $209 \cdot 7$ & 146 & 1.93 & 38 & 5 & $2 \cdot 19$ & 0.61 \\
\hline Fruits & $77 \cdot 4$ & 179 & $2 \cdot 72$ & 45 & 9 & 2.77 & 0.71 \\
\hline Chicken & $57 \cdot 6$ & 211 & 3.86 & 46 & 2 & $3 \cdot 71$ & 0.00 \\
\hline Candies & $38 \cdot 1$ & 236 & 4.02 & 47 & 4 & 4.68 & -0.11 \\
\hline Total meat & 142.5 & 120 & 2.75 & 9 & 0 & $5 \cdot 40$ & 0.24 \\
\hline Vegetables & $80 \cdot 2$ & 135 & $3 \cdot 25$ & 12 & 0 & 5.46 & 0.38 \\
\hline
\end{tabular}

VR, variance ratio.

* Percentage of individuals with 2-d mean intake equal to 0 .

† Percentage of individuals who did not report intake during the $20 \mathrm{~d}$.

‡ Spearman's correlation between probability of intake and amount eaten. 
(a)

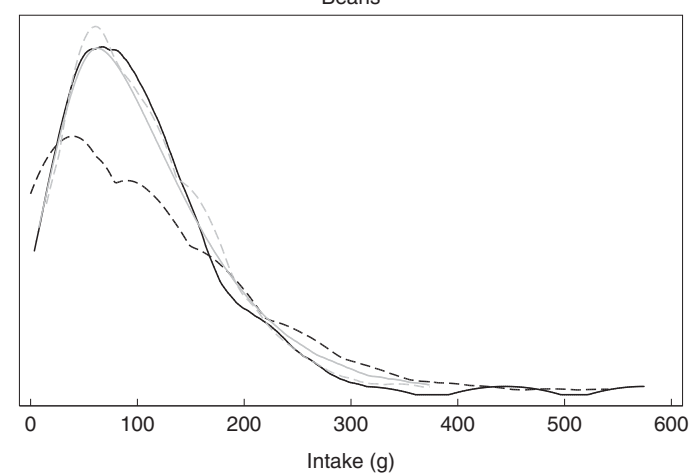

(c)

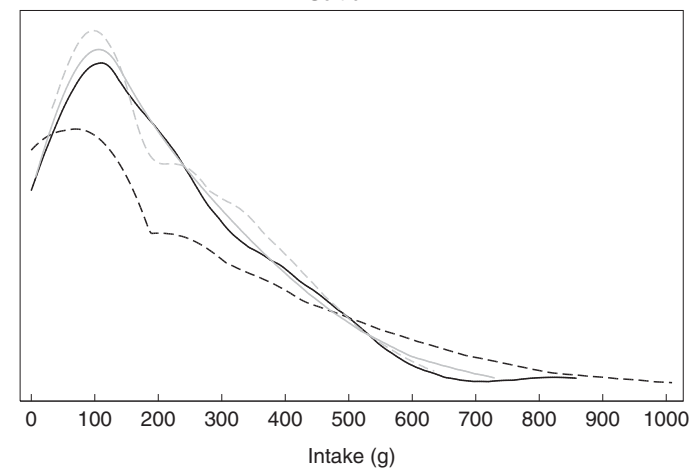

(e)

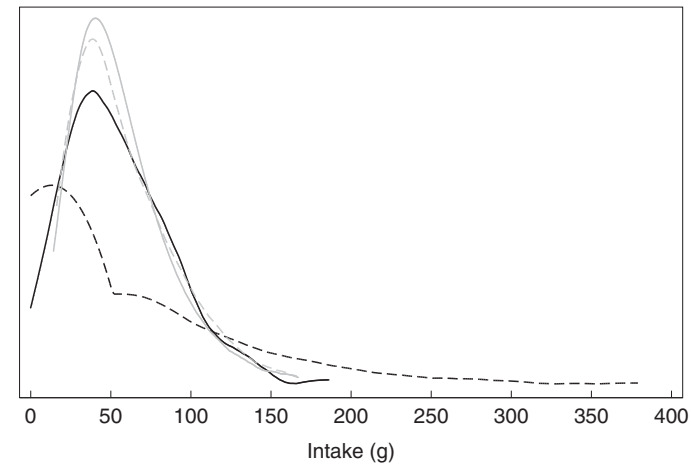

(g)

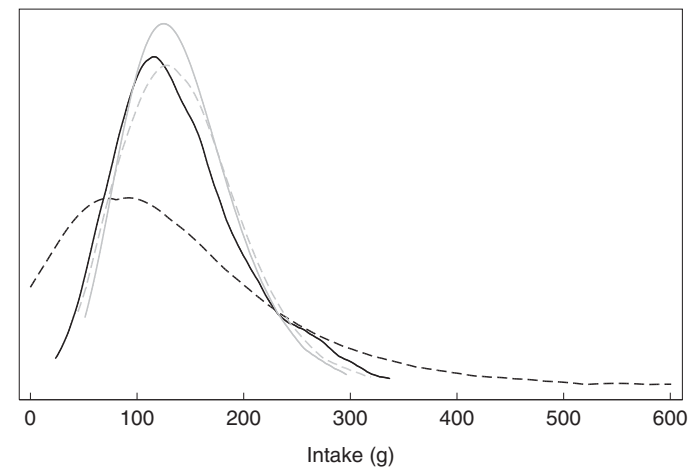

(b)

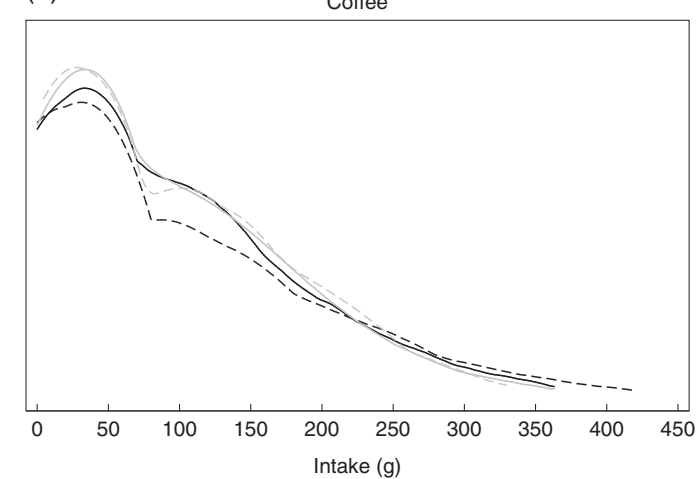

(d)

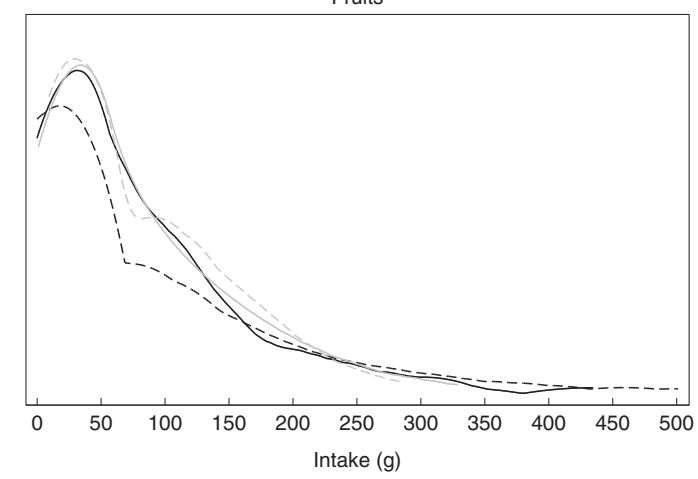

(f)

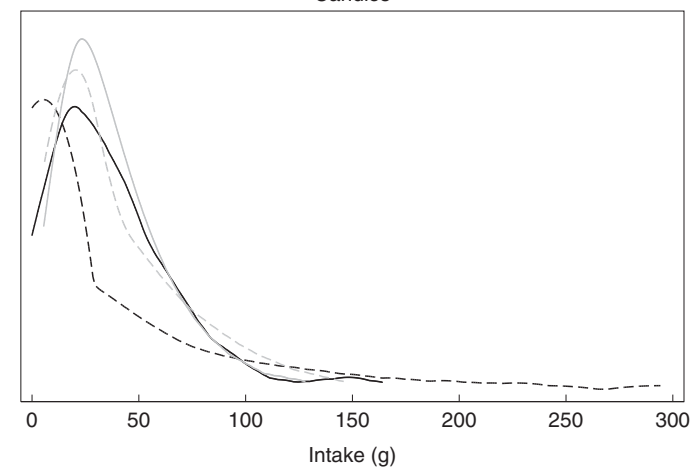

(h)

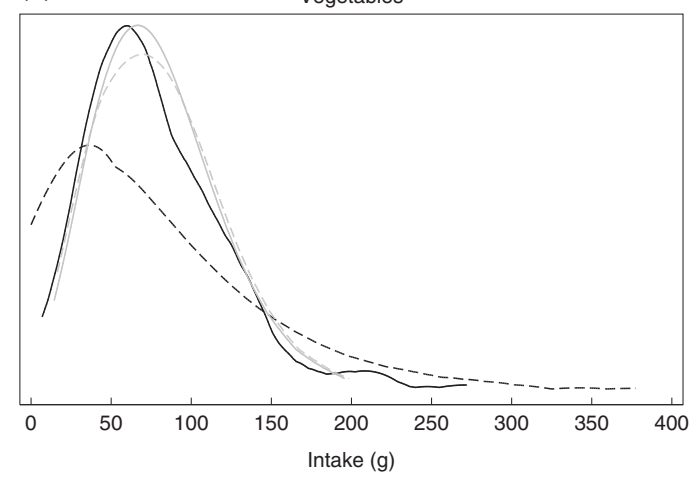

Fig. 1. (a-h) Food intake distributions from different methods: 2-d mean (- - - -), 20-d mean (- - , true intake), estimated from the Multiple Source Method (- - - ) and estimated from the National Cancer Institute $(\longrightarrow$ ) method. Distributions represent how close the estimated usual food intakes (using 2 collections days) are to the measured usual intakes. 2-d Means represent the uncorrected distribution based on a small number of collection days. 
(a)

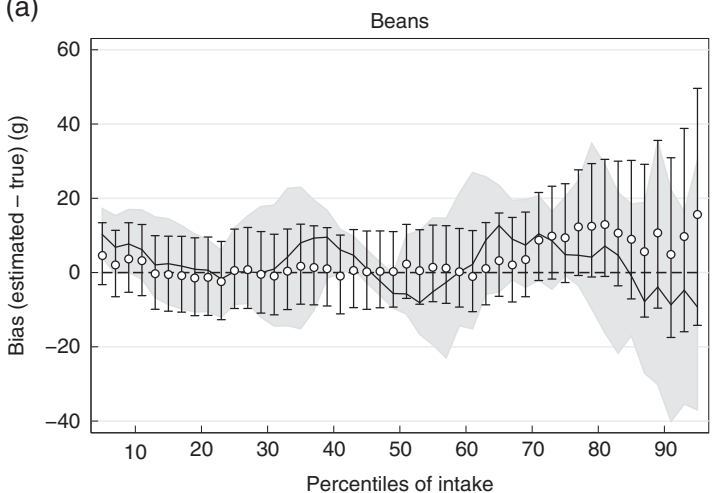

(c)

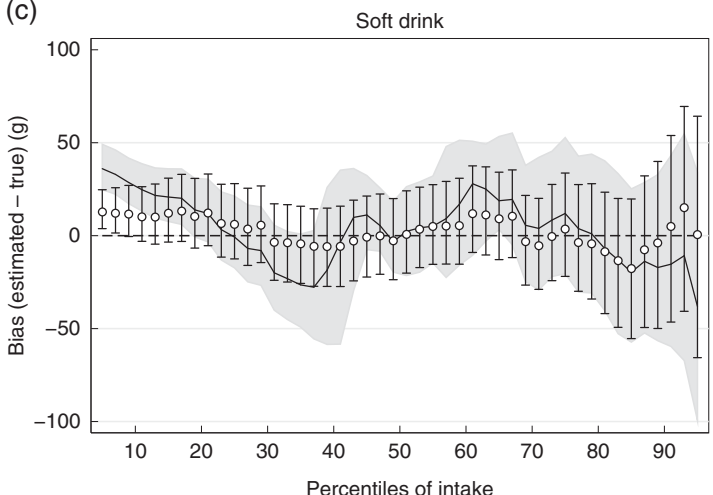

(e)

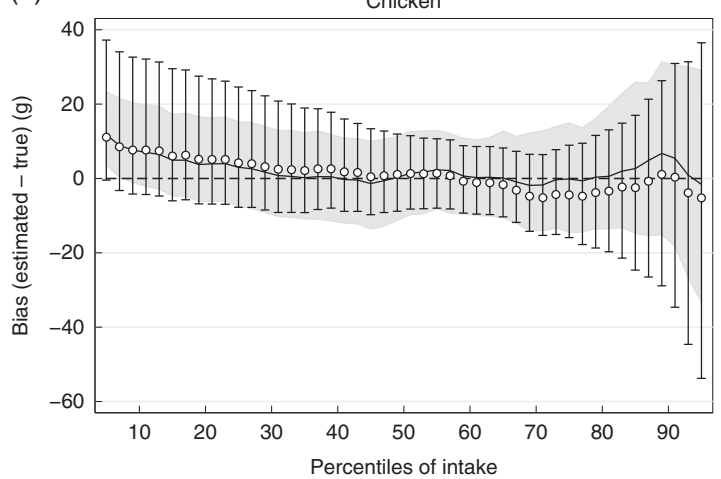

(g)

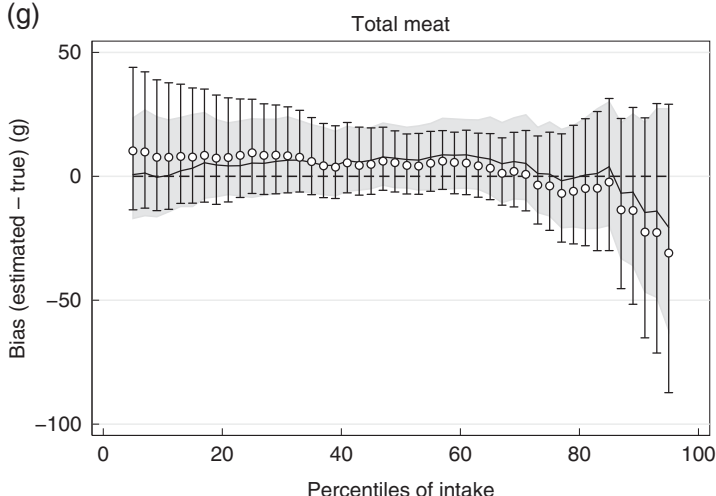

(b)

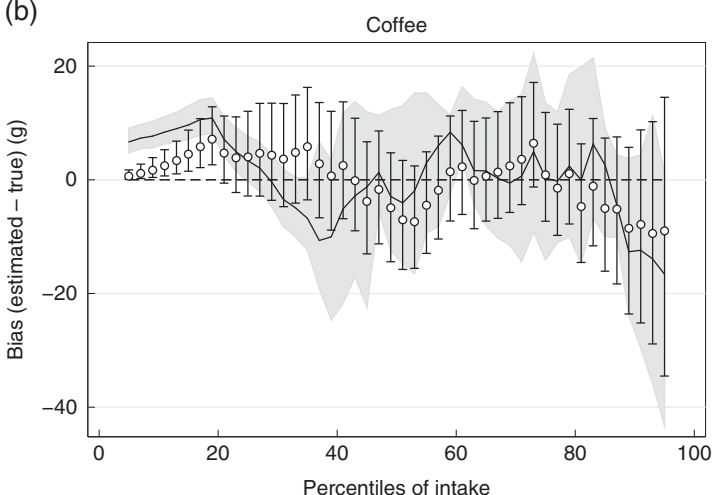

(d)
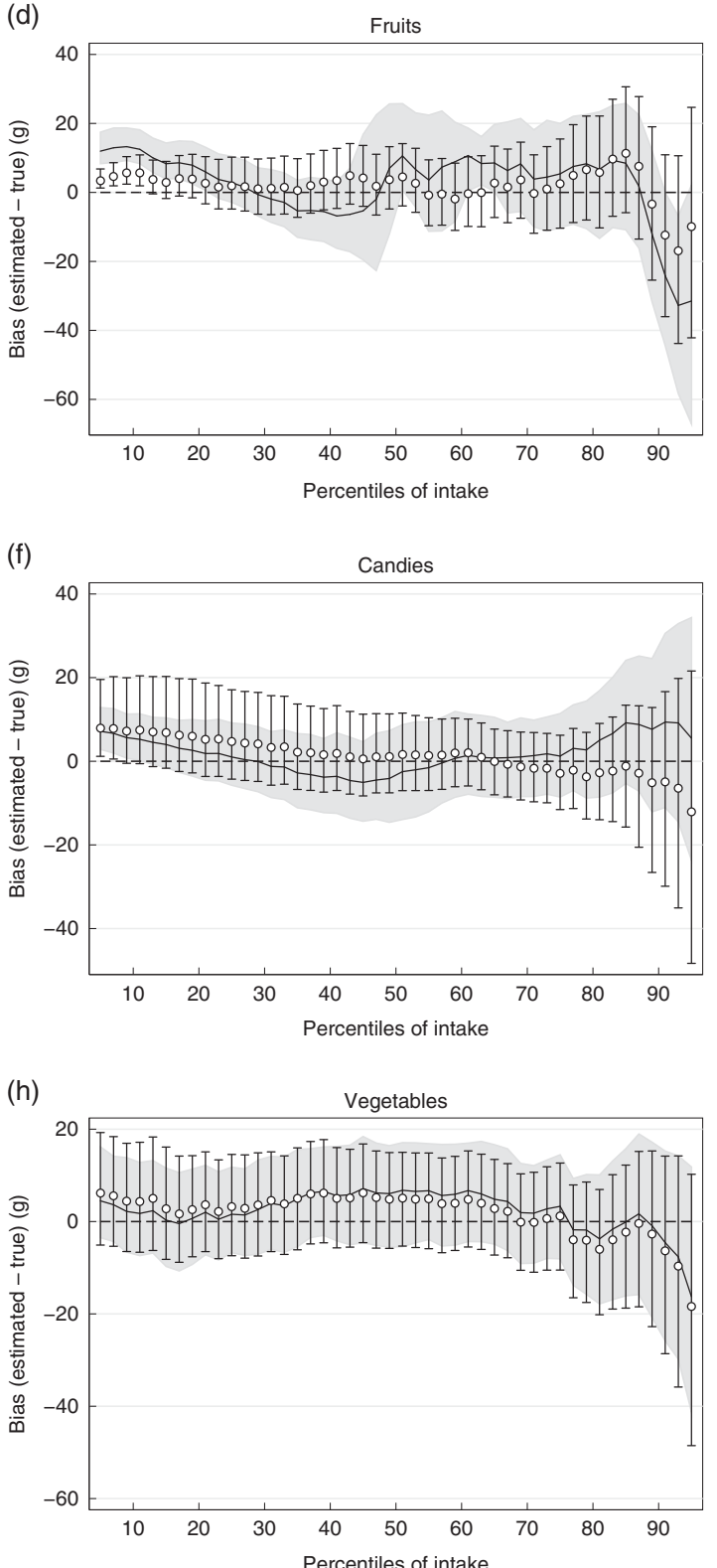

Fig. 2. (a-h) Bias and precision of the estimated percentiles of usual food intake. Biases were calculated as the absolute difference between the estimated and true intake. - and $\square$, Biases and precision regarding the Multiple Source Method (MSM); $\bigcirc$ and I, estimates regarding the National Cancer Institute (NCl) method. Figures show the over- and underestimation in each percentile from the MSM and $\mathrm{NCl}$ methods, and its variation when using different combinations of 2 collection days. 
(a)

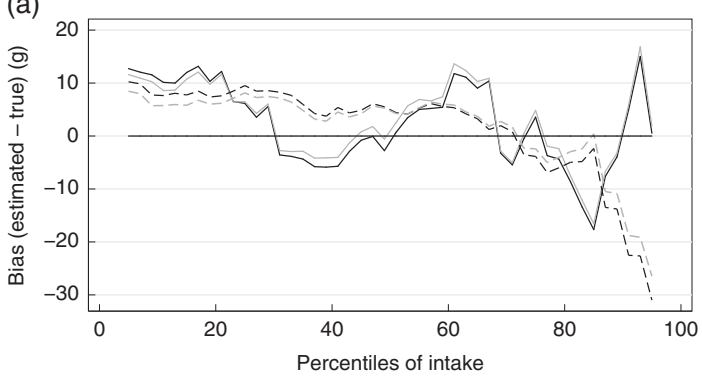

(b)

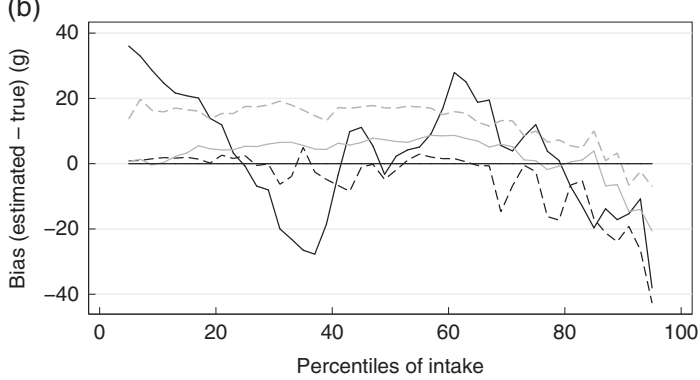

Fig. 3. Bias in the estimated percentiles of intake using 2 and $20 \mathrm{~d}$ compared with 20-d mean; (a) National Cancer Institute, (b) Multiple Source Method. Biases were

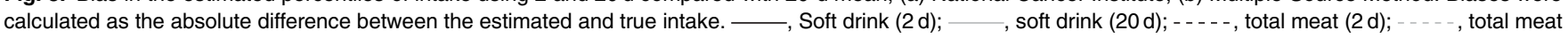
(20 d).

percentiles mainly for foods with high percentage of zero intake in a 2 -d combination, as in the case of soft drinks. In spite of the underestimation and overestimation of percentiles of intake, from a public health standpoint, these biases appear not to be of major concern. In general, the difference between true and estimated values was $<10 \%$ with higher variation in the low and upper percentiles. The most important implication of this error would be biased prevalence of inadequate intake when compared with a cut-off point ${ }^{(4)}$. Although there was a small precision for some estimated percentiles of intake, it happened mainly in the upper tail (see upper tail of distribution of fruits, soft drink and coffee). Subsequently, it does not substantially affect the proportion of a population above or below certain criteria of adequacy.

Our gold standard was the average of twenty replications of $24 \mathrm{HR}$ for each person. We assume that each $24 \mathrm{HR}$ is an unbiased measurement of the amount consumed on a given consumption day. It is clearly not true, and some studies have shown substantial systematic error when compared with biomarkers of energy, protein and $\mathrm{K}$ intakes ${ }^{(13)}$. Nonetheless, the methods used here correct only for within-person random error (day-to-day fluctuation in dietary intake) and not for systematic error, and it must be kept in mind when interpreting these results. The estimated distribution of usual intake is expected to reflect the actual between-person variance, but the mean intake and percentiles are usually underestimated because of systematic error ${ }^{(2,13)}$.

To be considered a satisfactory gold standard for the usual intake (in this case, free of within-person random error), two conditions must be met. First, the population mean must be stable through collection days. When collecting food intake information for many days, the population mean for each collection day has some variation due to expected random sample variation. The smaller the sample size, the higher the sample variation and smaller the precision of the mean for any specific day of intake. Despite the sample variation, population mean through collection days must keep stable - that is, with no trend of decrease as the number of collections increase. When this happens, it means that there is an additional source of error, probably due to some discouragement to keep answering thoroughly about food eaten by respondents. That is why the 1 st collection day is believed to be the one with the least systematic error, although there is no consistent evidence for that. Second, the variance must decrease and become stable - that is, the variance of intake measured by a single collection day per person is quite higher than that from the average of 5 collection days/person, and the latter is higher than that from 10 collection days. However, from a given number of collection days onwards, the variance becomes stable. The number of collection days required will vary according to the food item. These conditions were not met for some foods in this study and they were not included in our analysis (see online Supplementary Material).

A major limitation of this study is the small sample size, and in turn a small precision. However, this problem was mitigated by analysing all possible 2 collection day pairs and averaging the estimates so that extreme estimates, due to random sample variation, were cancelled out. Considering a classic study protocol in which two non-consecutive $24 \mathrm{HR}$ are administered, estimates from these statistical methods are more likely to be close to the true usual intake as found in this study, as long as the sample size provides enough precision. Otherwise, estimates from small samples would fall in a range of values such as those covered by the shaded area or vertical bars for each estimated percentile of intake. Other limitations inherent to the $24 \mathrm{HR}$ assessment, such as recall bias and interviewer bias, might have occurred, but we cannot know its extent as we did not use any biomarker.

We opted for not running the models by adjusting for covariates such sex, age and frequency of consumption. Although the correlation between frequency and amount would potentially improve the prediction, it has shown no effects on the estimation of the percentiles of usual food intake ${ }^{(9,14)}$. In fact, inclusion of frequency of consumption may not be beneficial when the interest is in the upper percentiles only ${ }^{(9)}$. Kipnis et al. ${ }^{(15)}$ proposed a regression calibration to correct for measurement error when testing the association between dietary intake and disease outcome and demonstrated by simulation that, in that case, frequency of consumption should be considered as an important predictor.

In conclusion, both methods seemed to be well suited for the estimation of percentiles of usual food intake, but with some increased bias in the lowest percentiles for foods with a high percentage of zero intake. In any case, the use of these methods mitigates an important limitation concerning the collection of long-term dietary intake in epidemiological studies, as a single replication of the $24 \mathrm{HR}$ would be enough to describe usual intake distribution in a population. 


\section{Acknowledgements}

This work was supported by the State of Rio de Janeiro Research Foundation (no. E-26/201.488/2014) and the Brazilian National Research Council (no. 481434/2013-5). The funders had no role in the design, analysis or writing of this article.

E. V.-J. conceptualised the study question, designed the study, analysed the data; D. C. R. S. O. supported the data management and data analyses; D. M. L. M. and R. M. F. reviewed the analyses and the manuscript and provided comments. E. V.-J. had primary responsibility for the final content. All the authors read and approved the final version of the manuscript.

There are no conflicts of interest.

\section{Supplementary material}

For supplementary material/s referred to in this article, please visit http://dx.doi.org/doi:10.1017/S0007114516002725

\section{References}

1. Nusser SM, Carriquiry AL, Dodd KW, et al. (1996) A semiparametric transformation approach to estimating usual daily intake distributions. J Am Stat Assoc 91, 1440-1449.

2. Freedman LS, Commins JM, Moler JE, et al. (2014) Pooled results from 5 validation studies of dietary self-report instruments using recovery biomarkers for energy and protein intake. Am J Epidemiol 180, 172-188.

3. Beaton GH, Milner J, Corey P, et al. (1979) Source of variation in 24-hour dietary recall data: implications for nutrition study design and interpretation. Am J Clin Nutr 32, 2546-2559.

4. Dodd KW, Guenther PM, Freedman LS, et al. (2006) Statistical methods for estimating usual intake of nutrients and foods: a review of the theory. J Am Diet Assoc 106, 1640-1650.

5. Tooze JA, Midthune D, Dodd KW, et al. (2006) A new statistical method for estimating the usual intake of episodically consumed foods with application to their distribution. $J \mathrm{Am}$ Diet Assoc 106, 1575-1587.

6. Hartig U, Haubrock J, Knüppel S, et al. (2011) The MSM program: web-based statistics package for estimating usual dietary intake using the Multiple Source Method. Eur J Clin Nutr 65 , S87-S91.

7. Barbosa FS, Sichieri R \& Junger WL (2013) Assessing usual dietary intake in complex sample design surveys: the National Dietary Survey. Rev Saúde Pública 47, 171s-176s.

8. Souverein OW, Dekkers AL, Geelen A, et al. (2011) Comparing four methods to estimate usual intake distributions. Eur J Clin Nutr 65, S92-S101.

9. Goedhart PW, van der Voet H, Knüppel S, et al. (2012) A comparison by simulation of different methods to estimate the usual intake distribution for episodically consumed foods. Supporting Publications 2012:EN-299. http://www.efsa.europa. eu/sites/default/files/scientific_output/files/main_documents/ 299e.pdf (accessed July 2016).

10. Freedman LS, Guenther PM, Krebs-Smith SM, et al. (2008) A population's mean healthy eating index-2005 scores are best estimated by the score of the population ratio when one 24-hour recall is available. J Nutr 138, 1725-1729.

11. Conway JM, Ingwersen LA, Vinyard BT, et al. (2003) Effectiveness of the US Department of Agriculture 5-step multiple-pass method in assessing food intake in obese and non obese women. Am J Clin Nutr 77, 1171-1178.

12. Verly E Jr, Castro MA, Fisberg RM, et al. (2012) Precision of usual food intake estimates according to the percentage of individuals with a second dietary measurement. $J$ Acad Nutr Diet 112, 1015-1020.

13. Kipnis V, Subar AF, Midthune D, et al. (2003) Structure of dietary measurement error: results of the OPEN biomarker study. Am J Epidemiol 158, 14-21.

14. Verly E Jr, Fisberg RM \& Marchioni DML (2013) Is the food frequency consumption essential as covariate to estimate usual intake of episodically consumed foods? Eur J Clin Nutr 66, 1254-1258.

15. Kipnis V, Midthune D, Buckman DW, et al. (2009) Modeling data with excess zeros and measurement error: application to evaluating relationships between episodically consumed foods and health outcomes. Biometrics 65, 1003-1010. 